\title{
AVOIDING NERVE DAMAGE DURING ELBOW ARTHROSCOPY
}

\author{
P. D. MARShall, J. A. FAIRClough, S. R. JOHNSON, E. J. EVANS \\ From Cardiff Royal Infirmary and the University of Wales, Cardiff, Wales
}

To define the anatomical relationships of the nerves to the common arthroscopy portals at the elbow an arthroscope was introduced into 20 cadaver elbows and the positions of the nerves were then determined by dissection.

In all cases the posterior interosseous nerve lay close to the radiohumeral joint and to the anterolateral portal. Pronation of the forearm displaced the nerve away from the arthroscope.

The median nerve passed consistently within $14 \mathrm{~mm}$ of the arthroscope when it was introduced through the anteromedial portal. The branches supplying the superficial forearm flexor muscles were at risk.

J Bone Joint Surg [Br] 1993; 75-B: 129-31.

Received 13 February 1992; Accepted 9 July 1992
Elbow arthroscopy is associated with recognised risks of injury to important peripheral nerves. Damage to the radial nerve as a complication of the anterolateral approach has been reported (Guhl 1985; Thomas, Fast and Shapiro 1987), and complications of the anteromedial approach have included median nerve damage (Andrews and Carson 1985) and traumatic neuroma of the medial cutaneous nerve of the forearm (Lynch et al 1986).

To find the precise relationships of the nerves to the arthroscopy portals in common use, an arthroscope was introduced into cadaver elbows and the position of the nerves was determined by dissection. We particularly studied the variations in the course of the posterior interosseous nerve since it is at risk during the use of the anterolateral portal. Although there have been some previous studies (Andrews and Carson 1985), none has detailed the variations in the course and origin of this nerve relative to the arthroscopy portal.

\section{MATERIALS AND METHODS}

We used 20 cadaver elbows and a $4 \mathrm{~mm}, 30^{\circ}$ angled arthroscope. The elbows were flexed to $90^{\circ}$ and the lateral and medial epicondyles, the radial head, and the tip of the olecranon were located by a surgical marker. Small

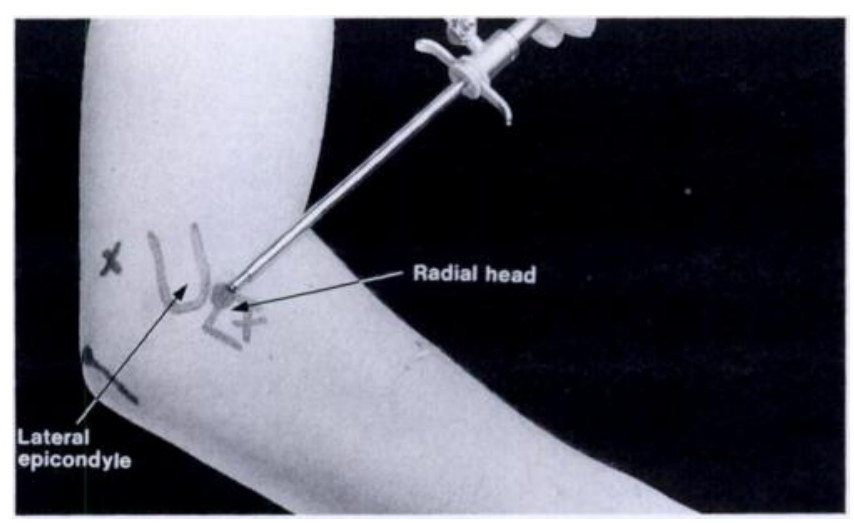

Fig. 1

Figure 1 - Left elbow showing the correct position for insertion at the anterolateral portal, directly into the anterior aspect of the radiohumeral joint. 
skin incisions followed by the use of a blunt trocar were used to establish the following portals:

Anterolateral. The arthroscope was introduced just anterior to the radial head, which was palpated while pronating and supinating the forearm. Although the site of introduction is often described as $3 \mathrm{~cm}$ distal and $1 \mathrm{~cm}$ anterior to the lateral epicondyle, adherence to these guidelines in a small elbow can result in the entry portal being positioned too distally, over the radial head itself (Fig. 1).

Anteromedial. The arthroscope was introduced $2 \mathrm{~cm}$ distal and $2 \mathrm{~cm}$ anterior to the medial epicondyle, aimed directly towards the centre of the elbow (Fig. 2).

Posterolateral. The arthroscope was introduced $3 \mathrm{~cm}$ proximal to the tip of the olecranon, just lateral to the triceps tendon (Fig. 3).

The major vessels and nerves were then dissected and transverse sections were taken through the elbow, to demonstrate their relationship to the arthroscopy portals (Figs 4, 5).

\section{RESULTS}

Using the anterolateral approach, the radial nerve was

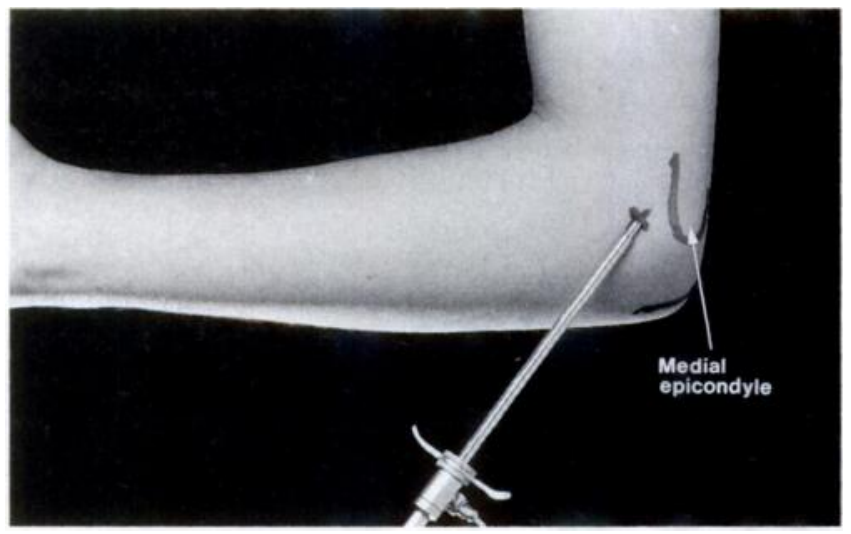

Fig. 2

Figure 2 - Left elbow showing the correct position for insertion at the anteromedial portal, $2 \mathrm{~cm}$ distal and $2 \mathrm{~cm}$ anterior to the medial epicondyle.

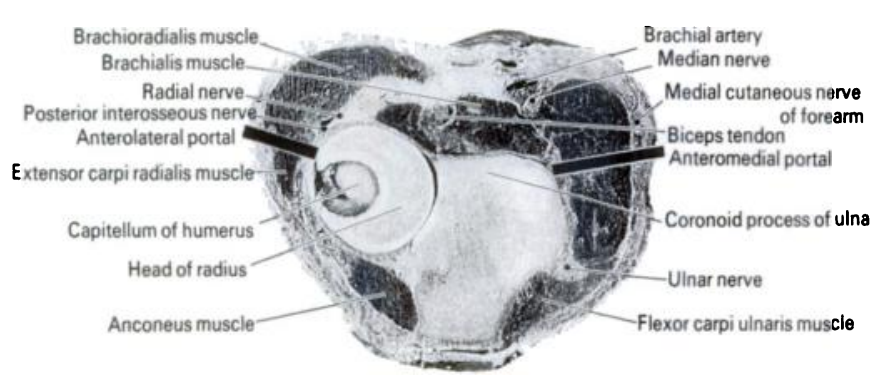

Fig. 4 found to pass consistently within $15 \mathrm{~mm}$ of the arthroscope. At greater risk was the posterior interosseous (deep) branch. In all 20 elbows, this nerve arose from the radial nerve as it passed between the brachialis and brachioradialis muscles, anterior to the lateral epicondyle of the humerus. Both nerves then passed anteromedial to the radiohumeral joint but, because of its deeper course, the posterior interosseous nerve lay closer to the joint than did the radial nerve (Fig. 4). Pronation of the forearm caused the posterior interosseous branch to be displaced anteromedially away from the radiohumeral joint, distancing it somewhat from the anterolateral portal. With the forearm in the mid-prone position (the normal position for arthroscopy through the anterolateral portal), the distance of the posterior interosseous branch from the anterolateral portal varied from 1 to $13 \mathrm{~mm}$ (mean $5 \mathrm{~mm}$, SD $3.1 \mathrm{~mm}$ ). We found that provided the instrument was inserted parallel to the joint it passed safely behind the posterior interosseous nerve. If, however, it was directed anteriorly or distally the nerve was at risk.

Using the anteromedial approach, the median nerve was found to pass consistently within $14 \mathrm{~mm}$ (mean $6.5 \mathrm{~mm}, \mathrm{SD} 3.3 \mathrm{~mm}$ ) of the arthroscope. The first branches

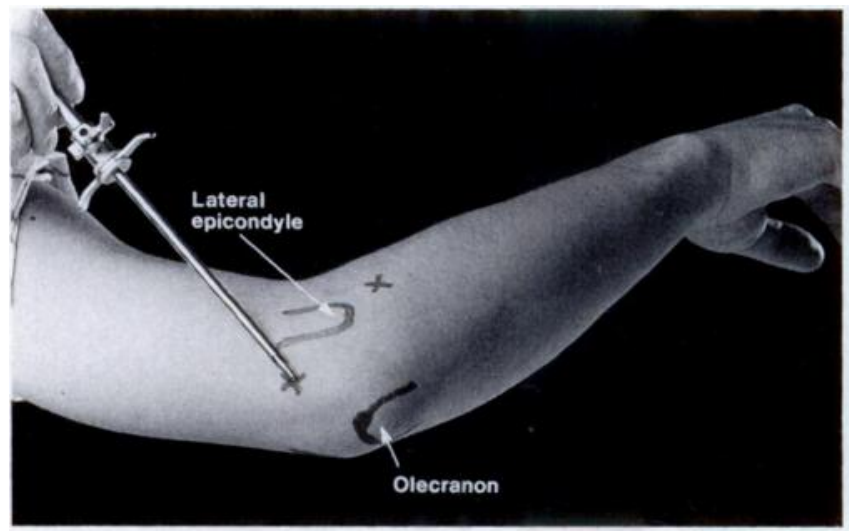

Fig. 3

Figure 3 - Left elbow showing the correct position for insertion at the posterolateral portal, $3 \mathrm{~cm}$ proximal to the tip of the olecranon, just lateral to the triceps tendon.

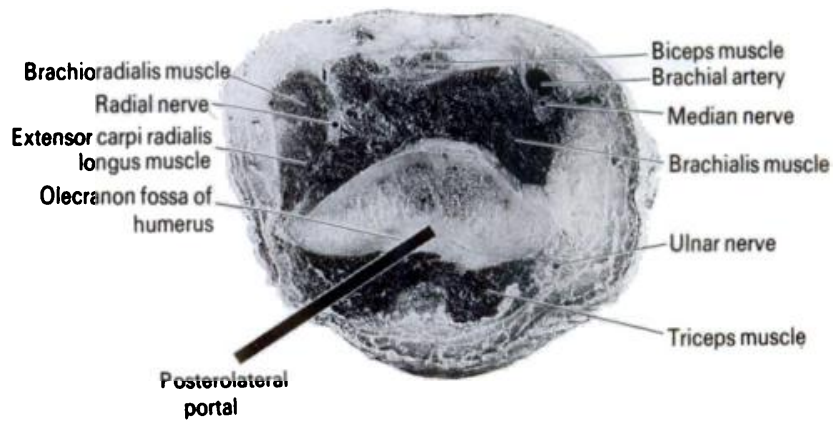

Fig. 5

Figure 4 - Transverse section through a right elbow at the level of the radiohumeral joint, looking proximally. The locations of the peripheral nerves relative to the anterolateral and anteromedial arthroscopic portals are shown. Figure 5 - Transverse section through a right arm, just proximal to the epicondyles of the humerus, looking proximally. The locations of the peripheral nerves relative to the posterolateral arthroscopic portal are shown. 
of the median nerve, lying on the anterior aspect of the brachialis muscle and supplying the superficial forearm flexor muscles, were at greater risk than their parent nerve, as they lay closer to the joint capsule. Injury to these branches could be avoided by passing the arthroscope directly anterior to the trochlea, thus ensuring that it did not pass anterior to the medial border of the brachialis muscle.

Also at risk during the anteromedial approach were branches of the medial cutaneous nerve of the forearm. These were found to lie in variable positions relative to the point of insertion of the arthroscope and it is not possible to give precise guidance on how to avoid injury. The risk of damaging the superficial cutaneous nerves, however, could be reduced by incising the skin in a longitudinal direction only to the level of the dermis. Subsequent blunt dissection to part the tissue planes reduces the risk of injury to underlying neurovascular structures.

Using the posterolateral portal we found little risk of neurovascular injury during the insertion of the arthroscope. Once in the joint, however, it was relatively easy to compress the ulnar nerve against the medial epicondyle by pushing the tip of the instrument medially.

\section{DISCUSSION}

The distances between the nerves and the arthroscopy portals are likely to be somewhat greater in vivo than those measured in the cadaver because of the greater water content of living tissues. Nevertheless, our study does show the relative risks to the different nerves.

These will be reduced if the joint is distended with fluid before insertion of the arthroscope. In vivo this has the effect of lifting the soft tissues and nerves away from the joint. Distension was attempted in the cadaver elbows, but could not be achieved because of the reduced elasticity of the embalmed tissues.

The correct entry point for the anterolateral portal is reported to be $3 \mathrm{~cm}$ distal and $1 \mathrm{~cm}$ anterior to the lateral epicondyle (Andrews and Carson 1985; Lynch et al 1986). We have found it more reliable, both in vivo and in our cadaver studies, to determine the site of entry by locating the position of the radial head while pronating and supinating the forearm, and then inserting the arthroscope directly into the anterior aspect of the radiohumeral joint. Figure 1 illustrates the correct position.

During the posterolateral approach injury to the ulnar nerve can be avoided by refraining from forceful manipulation of the instrument posterior to the medial epicondyle.

We wish to thank Mr K. Bellamy for his help and advice in the production of the illustrations used in this article.

No benefits in any form have been received or will be received from a commercial party related directly or indirectly to the subject of this article.

\section{REFERENCES}

Andrews JR, Carson WG. Arthroscopy of the elbow. Arthroscopy 1985; 1:97-107.

Guhl JF. Arthroscopy and arthroscopic surgery of the elbow. Orthopedics $1985 ; 8: 1290-6$.

Lynch GJ, Meyers JF, Whipple TL, Caspara RB. Neurovascular anatomy and elbow arthroscopy : inherent risks. Arthroscopy 1986; 2:190-7.

Thomas MA, Fast A, Shapiro D. Radial nerve damage as a complication of elbow arthroscopy. Clin Orthop 1987; 215:130-1. 